\title{
Which outcomes are reported in cellulitis trials? Results of a review of outcomes included in cellulitis trials and a patient priority setting survey
}

Running head: Outcomes in cellulitis treatment and prevention trials

Word count: 2874 (main body including headings)

Table count: 4

Figure count: 4

Dr Emma Smith, Centre of Evidence-based Dermatology, The University of Nottingham emma.smith111@nhs.net

Dr Mitesh Patel, Division of Primary Care, The University of Nottingham

Professor Kim Thomas, Co-director, Centre of Evidence-based Dermatology, The University of Nottingham

Funding sources/sponsors: The study was developed with support from the UK

Dermatology Clinical Trials Network (UK DCTN). The UK DCTN is grateful to the British Association of Dermatologists and the University of Nottingham for financial support of the Network

Conflicts of interest: None known

What is already known about this topic?

- Cellulitis can have a significant impact on the physical and psycho-social wellbeing of patients.

- There is currently no consensus on what outcomes should be measured in cellulitis trials. This makes it difficult to summarise treatment effects in meta-analyses.

What does this study add?

- This review is the first to combine and compare clinical outcomes in randomised controlled trials (RCTs) assessing treatment and prevention of cellulitis with outcome themes deemed important to patients and health care professionals.

- We have highlighted the disparity in clinical outcomes published in these RCTs and the lack of patient reported outcomes.

- Following the COMET initiative, we suggest that homogenous outcomes should be sought and specified for future research in cellulitis. 
Background: There is an emerging need to develop consistent outcomes in clinical trials to allow effective comparison of treatment effects. No systematic review has previously looked at the reporting of outcome measures used in randomised controlled trials (RCTs) on treatment and prevention of cellulitis (erysipelas).

Objectives: The primary aim of this review was to describe the breadth of outcomes reported from RCTs on cellulitis treatment and prevention. The secondary aim was to identify outcome themes from patient and health care professionals' feedback from a cellulitis priority setting partnership (PSP).

Methods: We conducted a review of all outcome measures used in RCTs from two recent Cochrane reviews. Free text responses from a cellulitis priority setting survey were used to understand the perspectives of patients and healthcare professionals.

Results: Outcomes from 42 RCTs on treatment of cellulitis and six RCTs on prevention of cellulitis were reviewed. Only 28 trials stated their primary outcome. For trials assessing treatment of cellulitis, clinical response to treatment was categorised in 25 different ways. Five of these trials used an outcome that was in accordance with FDA guidance and only four trials incorporated either quality of life or patient satisfaction. For trials assessing prevention of cellulitis, recurrence was the key outcome measure. From the cellulitis PSP, prevention of recurrence, clinical features and long-term disease impact were the most important outcome themes for patients.

Conclusions: We have shown that in cellulitis treatment and prevention research, there is significant heterogeneity in clinical outcomes, inadequate focus on patient-reported outcomes, and a disparity between what is currently measured and what patients and healthcare professionals feel is important. We recommend that future cellulitis treatment trials consider the use of longer-term outcomes to capture recurrence and long-term morbidity, as well as short-term resolution of acute infection.

Keywords: cellulitis, erysipelas, outcomes, core outcome set, priority
setting partnership Introduction 
Cellulitis is an acute, potentially serious bacterial infection of the cutaneous and subcutaneous tissue, usually occurring on the lower limb. The most common causative organism is group A streptococcus (streptococcus pyogenes) ${ }^{1}$ which causes a spreading area of erythema, tenderness and swelling. The illness can be complicated by ulceration, purulence and progression to systemic disease in severe cases. Erysipelas is characterised by a superficial, well-demarcated area of inflammation, whilst cellulitis often extends to the subcutaneous tissue ${ }^{2}$. It is often difficult to distinguish between them clinically; therefore for the purpose of this review we have used cellulitis as the umbrella term for cellulitis, erysipelas and skin and soft tissue infection, which reflects current practice.

Cellulitis poses a significant health burden to the NHS: during 2014-15 in England, 114,190 patients were treated in hospital for cellulitis with a median length hospital stay of 6 days $^{3}$. In addition to short-term signs and symptoms, the disease can have a significant long-term impact on patient's physical and mental health ${ }^{4}$. In certain groups of patients there are high levels of recurrence of cellulitis arising from repeated damage to the lymphatic system and the presence of certain risk factors ${ }^{2}$.

Despite the impact of the disease, there are few well-conducted trials looking into the treatment and prevention of cellulitis ${ }^{5,6}$. It has been reported that clinical assessment of cellulitis many not capture the patient's experience, and may be unreliable in reflecting real effects for patients ${ }^{7}$.

Defining standard, measurable outcomes is essential for well-designed, robust clinical trials, to provide trial results can be reliably compared ${ }^{8}$. Outcomes need to be meaningful and relevant for service-users, including patients and clinicians, in order to make a long-term difference to future practice.

It has been recognised that many outcomes in clinical trials lack sufficient validation and there is support for development of core outcome sets (COS) for trials to address this issue, as proposed by the COMET initiative ${ }^{8}$. A COS is an agreed set of outcomes that should be measured and reported, as a minimum, in all clinical trials in a specific area of health care ${ }^{9}$. The initial stage in developing a core outcome set is to identify what outcomes are available, and to establish which are most important and relevant for healthcare users ${ }^{10,11}$.

The Food and Drug Authority (FDA) have attempted to address the lack of consistent outcomes for clinical trials of Acute Bacterial Skin and Soft tissue infections (ABSSIS). In 2013 they updated their guidance on endpoints in such trials which states the primary outcome should be: \% reduction in lesion size, measured at 48 to $72 \mathrm{~h}$ compared to baseline ${ }^{12}$. However, this recommendation is largely based on historical trial data dating to pre-antibiotic era and has undergone incomplete validation ${ }^{13}$.

There have been no systematic reviews assessing outcome measures used in cellulitis trials. The main aim of this review was to describe all the outcome measures reported in randomised control trials (RCTs) on the treatment and prevention of cellulitis. The secondary aim was to identify outcome themes from patient and health care professionals' feedback from a cellulitis priority setting partnership (PSP) ${ }^{14}$.

\section{Methods}




\section{Study 1: Review of outcome measures in RCTs for the treatment and prevention of cellulitis}

\section{Search strategy}

This review included all RCTs that were included in two Cochrane reviews: Interventions for the prevention of recurrent erysipelas and cellulitis ${ }^{5}$, published in June 2017, and the Cochrane update: Interventions for the treatment of cellulitis and erysipelas (awaiting publication but obtained from personal communication with authors). Studies that assessed treatment or prevention of cellulitis, erysipelas or skin and soft-tissue infection were included in this review. A search of COMET and PROSPERO was performed to ensure that no existing core outcome initiatives of thematically similar reviews were already registered.

Data regarding treatment complications, adverse events and side effects were not collected as this data is routinely collected in drug trials was not an aim of this review.

The study protocol for this review is available to view on the Centre of Evidence Based Dermatology website ${ }^{15}$

\section{Data extraction and assessment of bias}

Reported outcomes were extracted using a standardized template that was piloted prior to use. If the outcome measures were not clearly stated in the methods section of the trial report, but were described in the results section, then these were included.

Details of outcome measures were extracted from each paper by two independent researchers (ES and MP). Any disagreements were resolved by two further independent researchers.

Data extracted were: (i) demographics of trial (author and date of publication) (ii) trial type: treatment/ prevention of cellulitis, drug/ non-drug, (iii) whether primary outcome was stated (iv) total number of outcomes per trial (v) whether outcomes were in line with FDA guidance on skin infections (vi) additional comments on outcomes used in trials e.g. justification of outcomes (vii) Broad outcome domains: clinical, microbiological, biochemical, treatment-related, patient-focused, additional outcomes. For each outcome domain, further definitions were used for the specific outcomes measured e.g. cure/failure/response, length of hospital stay, quality of life. Additionally, for clinical outcome, the specific clinical features that were assessed as part of the outcome were identified e.g. erythema, swelling, warmth (viii) definition of each outcome (ix) how the outcome was assessed $\mathrm{ix}$ ) scales used (xi) outcome assessor e.g. nurse/clinician/patient (xii) Timing/frequency of assessment.

Storage and analysis of all the data was undertaken by the lead researcher (ES) using Microsoft Excel, 2010, CEBD. Data are presented descriptively, and results reported separately for cellulitis treatment trials and prevention trials.

\section{Study 2: Understanding patient and healthcare professionals' perspectives}

Using free text data collected during a Priority Setting ${ }^{14}$ we sought to identify outcomes of importance to patients and healthcare professionals. Responses were submitted by 401 survey participants (171 patients/carers, 217 healthcare professionals, 13 other). Participants were asked the following: "What questions about the diagnosis, treatment or prevention of cellulitis would you like to see answered by research?" Data relevant to outcome measurement were extracted from the free text responses and used to identify key outcome themes using the word repetition technique ${ }^{16}$. 


\section{Results}

From the two Cochrane reviews, 48 trials were identified and included in the final analysis. This included 42 RCTs assessing acute treatment of cellulitis and six RCTs assessing prevention of cellulitis. No studies were excluded. The total number of outcomes measured per trial ranged from one to eight outcomes, across six different outcome domains (Figure $1)$. Only $28(58 \%)$ trials stated the primary outcome. Two papers assessed nonpharmacological treatments for cellulitis; one reviewed the use of vibration therapy and the other an alternative therapy: sodium selenite. Six trials evaluated treatment strategies for skin and soft-tissue infections but did not analyse results for cellulitis separately. However, data from these papers were included in this review, to maximise capture of outcomes.

\section{RCTs assessing treatment of cellulitis}

\section{Clinical outcomes}

All trials assessed at least one clinical outcome. Of the RCTs assessing treatment of cellulitis, clinical response was categorised according to a range of definitions (Table 1) with the majority of papers classifying response according to "cure", "failure" or "improvement". The timing of the clinical assessment was variable. Some recent trials assessed clinical response at an early time-point, 2-3 days after treatment initiation. However, most trials assessed response at a "test-of-cure (TOC)" visit, which ranged from 2 to 42 days after the end of treatment (Table 1). A successful clinical response or "cure" was defined most commonly as either "complete resolution of presenting signs and symptoms", or "resolution of the infection to an extent that no further antibiotic therapy was required" (Table 2 ). Four (10\%) trials included "absence of recurrence" of infection as part of the definition of a successful clinical response.

There were 10 different signs and symptoms of infection used to describe clinical response across the trials (Figure 2 ). The most commonly assessed clinical feature was erythema. Of 34 trials that assessed erythema, six (18\%) assessed this numerically, measuring the diameter or area of erythema, six (18\%) graded the erythema on a severity scale (Figure 3); and 13 (38\%) used "clinical assessment/ evaluation" without further detail of the methods used.

Sixteen (38\%) of trials assessed at least one biochemical marker as part of clinical response to treatment. Of these, the most commonly assessed markers were white cell count, erythrocyte sedimentation rate and c-reactive protein.

\section{Microbial outcomes}

Twenty-four (57\%) trials included a microbial outcome, assessed using either blood or wound culture. The majority of trials categorised microbial response according to complete/presumed eradication, persistence/presumed persistence and indeterminate/ not evaluable (Table 4). Of the trials assessing a microbial response, the timing and frequency of assessment was either not stated or unclear in $18(42 \%)$ trials.

\section{Treatment-related outcomes}

Sixteen (38\%) trials assessed a treatment-related outcome. These included: duration of antibiotic treatment (13), number of doses of antibiotic (2) and serum antibiotic concentration (1). One trial used "number of doses of antibiotic until clinical response" as the primary outcome for the study. 
Four trials included patient-focused outcomes in their methodology. One trial assessed Quality of Life (QoL) using the EuroQol-5 dimensions (EQ-5D) questionnaire. Two assessed patient's impression of improvement of cellulitis: one using the patient Global Impression of Improvement scale and one using predefined categorical statements: improved, stayed the same, worsened. One paper assessed patient satisfaction with treatment received, assessing each of the parameters: convenience, effectiveness and overall satisfaction on a scale of $0-4$. Eleven (26\%) trials assessed patient-reported pain (Table 3).

\section{FDA guidance}

Five $(12 \%)$ trials assessing treatment of cellulitis that were published from 2011 onwards had their primary outcome in line with FDA guidance.

\section{RCTs assessing prevention of cellulitis}

The primary outcome of trials assessing prevention of cellulitis was either: number of episodes of recurrence or time to recurrence of cellulitis for all six RCTs ${ }^{17-22}$. Three $(50 \%)$ of the trials specified the clinical features to be used in assessing an episode of recurrence. Follow-up time varied from 3 months to 3 years. Two (33\%) papers explained how patients were monitored during the follow-up period: via routine telephone calls, 3-monthly during the treatment phase and 6 monthly during the follow-up phase It is worth noting that the PATCH I and II trials intended to collect data on the impact of cellulitis on QoL, however this was abandoned due to technical difficulties around timing of assessments in recurrent episodes ${ }^{21}$.

\section{Additional outcomes}

Additional outcomes measured in all trials (assessing both treatment and prevention of cellulitis) included: length of hospital stay (ten trials) and cost-related outcomes (five trials).

\section{Outcome themes from the cellulitis PSP}

A total of 846 uncertainties were submitted during the PSP survey. These data were reviewed for outcome themes, and $254(30 \%)$ responses included reference to outcomes of importance to participant. From these responses, 263 outcome themes were identified (some responses contained more than 1 outcome theme), of which 73 (28\%) were from patients and $190(72 \%)$ from healthcare professionals. Prevention of recurrence was the most frequently stated outcome theme of importance from both patients and healthcare professionals (Figure 4). "Treatment-focused" outcomes such as: length of acute and prophylactic antibiotic therapy and the use of objective markers to assess treatment response, were also key priorities for healthcare professionals. Assessment of clinical features (erythema, swelling and pain most commonly) and long-term morbidity/mortality were the next most important outcomes for patients. Surprisingly, only $2 \%$ of all outcome themes extracted focused on patient-orientated factors such as quality of life and treatment satisfaction.

\section{Discussion}




\section{Main findings}

\section{Variability in clinical outcomes}

This review highlights the significant variation of outcomes which are currently used in cellulitis trials, suggesting that efforts to streamline and develop a consensus-driven core outcome set would be valuable.

Clinical response to treatment was categorised in 25 different ways in the 42 treatment trials, and "cure"/ "success" or "resolution", had 18 different definitions that were measured over a range of timescales, from day 2 to day 35 after the end of treatment period. The majority of trials defined "cure" according to complete or partial resolution of presenting signs and symptoms of infection. However, the specific signs and symptoms of infection assessed, such as erythema, swelling and warmth were often poorly defined. Up to 10 different signs and symptoms of infection were each used by at least one trial to assess treatment response. This reflects the general lack of consensus in outcome assessment for cellulitis.

Some of the more recent trials used "early clinical response" as the primary outcome, defined as: "cessation of lesion spread (measured as the product of the length $x$ width of lesion) 48-72 hr after treatment initiation". Assessing treatment response at this earlier time-point may increase reliability of the measurement of drug effect, because the outcome is not confounded by natural improvement of disease ${ }^{23}$. Moreover, this endpoint is in line with the 2013 FDA guidance recommending early clinical response as the primary outcome ${ }^{12}$.

\section{I nadequate patient orientated outcomes}

Patient-focused outcomes such as patient-reported pain, treatment satisfaction and quality of life were rarely reported. We know that cellulitis has a significant impact on physical and psychological health, as well as activities of daily living and quality of life ${ }^{4}$, yet this significance is not reflected in the outcomes from the cellulitis trials published to date. Importantly, the survey results suggest that recurrence and long-term disease impact are important aspects to patients. To date, treatment trials have tended to be of short duration and focus on resolution of symptoms associated with the acute episode. This is not surprising given the cost and methodological complexities associated with longer-term trials, but may require greater consideration when planning future studies.

There were far more treatment trials than prevention trials, which again suggests an imbalance favoring short-term outcomes. More trials that address the prevention of longterm morbidity and recurrence are needed, so that treatment strategies can be developed that have a long-term beneficial impact for patients.

In contrast to patient responses, the survey results revealed the most important outcomes to healthcare professionals were: objective markers of disease response, length of antibiotic treatment and prophylaxis (in addition to recurrence). This is perhaps not surprising, given that healthcare professionals are responsible for both prescribing and objective assessment of treatment effect, rather than a subjective experience of disease.

\section{Limited use of microbial outcomes}

The use of blood or wound cultures in cellulitis management is debatable. Microbial outcomes are a poor indicator of treatment success as their yield is often low, even in the presence of infection ${ }^{24}$ and can be adversely affected if patients have been pre- 
treated with antibiotics. Furthermore, microbial cultures do not correlate well with severity of signs and symptoms or patient experience. ${ }^{24}$. This outcome measure is also of limited relevance to patients. However, the increasing incidence of antimicrobial resistance means that other indicators of resistance, such as the incidence and severity of other bacterial infections, may be important to collect.

\section{Strengths and weaknesses}

This review summarised the breadth of outcomes reported in published RCTs and analysed these alongside outcomes considered important by patients and healthcare professionals.

Since data on the views of patients and healthcare professionals were originally collected for another purpose (defining the research agenda for cellulitis research), they may not be as reflective of the results as if respondents had been asked specifically about research outcomes. Further qualitative work is required to replicate our findings and to full ascertain the perspectives of all key stakeholders.

The quality of included studies was not assessed as this was not the aim of this review, however many studies were poorly reported, making it difficult at times to establish a detailed understanding of the outcome measures used.

Due to time and resource limitations, included studies were identified from two recently updated Cochrane reviews. These were chosen because they are both up-to-date and sufficiently broad (covering any "treatment" and "prevention" of cellulitis) to gain the breadth of outcome data we aimed to capture. It is possible that more recent RCTs on cellulitis treatment and prevention have since been published.

\section{Generalisability}

The included trials were conducted in eight single countries and nine in multiple countries. This review captures a representative snapshot of existing outcomes measures used in cellulitis research. Others have highlighted similar variability in outcome measure instruments used in skin diseases such as eczema ${ }^{25}$, vitiligo ${ }^{26}$ and acne ${ }^{27}$.

\section{Conclusion}

Outcome measures for cellulitis should not only be consistent, to allow clinical trials to be adequately compared, but should be reflective of real impact in patient's day to day lives. This review has highlighted that in cellulitis research currently there is a lack of consensus over what should be measured, how that should be measured and over what time-frame. Future research should work towards validating current outcomes with a view to developing a COS for cellulitis. I deally this would include patient-reported outcomes and a long-term outcome measure, such as recurrence, to account for the chronicity of the disease that is important to many patients. Currently, researchers should seek to ensure outcomes are in line with FDA guidance specific to acute bacterial skin and soft tissue infections.

\section{Acknowledgments}

We would like to thank the researchers involved in the cellulitis PSP: R. Brindle, J.R. Chalmers, B. Gamble, N.A. Francis, D. Hardy, J. Hooper, V. Keeley, N.J. Levell, M. McPhee, L. Metcalf, M. Santer, M. Tarpey, P. Smart, A. Wallace and S. Wilkes for 
providing the survey data; the Cochrane skin group for help in accessing the clinical trials and Shelley Dowey for help with data extraction.

\section{References}

$1 \quad$ Bruun T, Oppegaard O, Kittang BR et al. Etiology of Cellulitis and Clinical Prediction of Streptococcal Disease: A Prospective Study. Open forum infectious diseases 2016; 3: ofv181.

2 Chlebicki MP, Oh CC. Recurrent cellulitis: risk factors, etiology, pathogenesis and treatment. Current infectious disease reports 2014; 16: 422.

3 Hospital Episode Statistics, Admitted Patient Care, England 2014-15. In: Microsoft Excel (Hopsital Episode Statistics APC, England-2014-15: Diagnosis [.xlsx], ed).

4 Carter K, Kilburn S, Featherstone P. Cellulitis and treatment: a qualitative study of experiences. British journal of nursing (Mark Allen Publishing) 2007; 16: S224, S6-8.

5 Dalal A, Eskin-Schwartz M, Mimouni D et al. Interventions for the prevention of recurrent erysipelas and cellulitis. The Cochrane database of systematic reviews 2017; 6: CD009758.

$6 \quad$ Kilburn SA, Featherstone P, Higgins B et al. Interventions for cellulitis and erysipelas. The Cochrane database of systematic reviews 2010: Cd004299.

7 Powers JH, 3rd, Das AF, De Anda C et al. Clinician-reported lesion measurements in skin infection trials: Definitions, reliability, and association with patientreported pain. Contemporary clinical trials 2016; 50: 265-72.

8 Kirkham JJ, Gorst S, Altman DG et al. COS-STAR: a reporting guideline for studies developing core outcome sets (protocol). Trials 2015; 16: 373.

9 Williamson PR, Altman DG, Blazeby JM et al. Developing core outcome sets for clinical trials: issues to consider. Trials 2012; 13: 132.

10 Schmitt J, Apfelbacher C, Spuls PI et al. The Harmonizing Outcome Measures for Eczema (HOME) roadmap: a methodological framework to develop core sets of outcome measurements in dermatology. The Journal of investigative dermatology 2015; 135: 24-30.

11 Williamson PR, Altman DG, Bagley $\mathrm{H}$ et al. The COMET Handbook: version 1.0. Trials 2017; 18: 280.

12 US Department of Health and Human Services FDA, Center for Drug Evaluation and Research (CEDR). Guidance for Industry, Acute Bacterial Skin and Skin Structure Infections: Developing Drugs for Treatment In. New HampshireUSA. 2013.

13 Talbot GH, Powers JH, Hoffmann SC. Developing Outcomes Assessments as Endpoints for Registrational Clinical Trials of Antibacterial Drugs: 2015 Update From the Biomarkers Consortium of the Foundation for the National Institutes of Health. Clinical infectious diseases : an official publication of the Infectious Diseases Society of America 2016; 62: 603-7.

14 Thomas KS, Brindle R, Chalmers JR et al. I dentifying priority areas for research into the diagnosis, treatment and prevention of cellulitis (erysipelas): results of a James Lind Alliance Priority Setting Partnership. The British journal of dermatology 2017.

15 Centre of Evidence Based Dermatology >Protocol Registration> Which outcomes should we measure in cellulitis trials? Results of a systematic review of ouctomes used in cellulitis trials and a patient priority setting survey [ONLINE] Available at: http://www. nottingham. ac.uk/research/groups/cebd/resources/protocolregistration.aspx. [Accessed 25 August 2017]. In.

16 Strauss C. In: What makes Tony run? Schemas as motive reconsideration in human motives and cultural models (R. D'Andrade CS, ed). Cambridge, UK: Cambridge University Press. 1992; 191-224. 
17 Chakroun M B-RF, Battikh R, et al. Benzathine penicillin prophylaxis in recurrent erysipelas. Med Mal Infect 1994; 24: 894-7.

18 Kasseroller R. Sodium Selenite as Prophylaxis Against Erysipelas in Secondary Lymphoedema. Anticancer Research 1998; 18: 2227-30.

19 Kremer M, Zuckerman R, Avraham Z et al. Long-term antimicrobial therapy in the prevention of recurrent soft-tissue infections. Journal of Infection 1991; 22: 3740.

20 Sjoblom AC, Eriksson B, Jorup-Ronstrom C et al. Antibiotic Prophylaxis in Recurrent Erysipelas. Infection 1993; 21: 390-3.

21 Thomas K, Crook A, Foster K et al. Prophylactic antibiotics for the prevention of cellulitis (erysipelas) of the leg: results of the UK Dermatology Clinical Trials Network's PATCH II trial. The British journal of dermatology 2012; 166: 169-78.

22 Thomas KS, Crook AM, Nunn AJ et al. Penicillin to prevent recurrent leg cellulitis. The New England journal of medicine 2013; 368: 1695-703.

23 Bruun T, Oppegaard O, Hufthammer KO et al. Early Response in Cellulitis: A Prospective Study of Dynamics and Predictors. Clinical infectious diseases : an official publication of the Infectious Diseases Society of America 2016; 63: 103441.

24 Van Daalen FV, Kallen MC, Van den Bosch CMA et al. Clinical condition and comorbidity as determinants for blood culture positivity in patients with skin and soft-tissue infections. European journal of clinical microbiology \& infectious diseases : official publication of the European Society of Clinical Microbiology 2017.

25 Schmitt J, Langan S, Williams HC. What are the best outcome measurements for atopic eczema? A systematic review. The Journal of allergy and clinical immunology 2007; 120: 1389-98.

26 Eleftheriadou V, Thomas KS, Whitton ME et al. Which outcomes should we measure in vitiligo? Results of a systematic review and a survey among patients and clinicians on outcomes in vitiligo trials. The British journal of dermatology 2012; 167: 804-14.

27 Barratt $\mathrm{H}$, Hamilton $\mathrm{F}$, Car J et al. Outcome measures in acne vulgaris: systematic review. The British journal of dermatology 2009; 160: 132-6.

28 Bernard $\mathrm{P}$, Plantin $\mathrm{P}$, Roger $\mathrm{H}$ et al. Roxithromycin versus penicillin in the treatment of erysipelas in adults: a comparative study. The British journal of dermatology 1992; 127: 155-9.

29 Bernard P, Chosidow O, Vaillant L. Oral pristinamycin versus standard penicillin regimen to treat erysipelas in adults: randomised, non-inferiority, open trial. BMJ (Clinical research ed.) 2002; 325: 864.

30 Tarshis GA, Miskin BM, Jones TM et al. Once-daily oral gatifloxacin versus oral levofloxacin in treatment of uncomplicated skin and soft tissue infections: doubleblind, multicenter, randomized study. Antimicrobial agents and chemotherapy 2001; 45: 2358-62.

31 O'Riordan W, Mehra P, Manos $\mathrm{P}$ et al. A randomized phase 2 study comparing two doses of delafloxacin with tigecycline in adults with complicated skin and skinstructure infections. International journal of infectious diseases : IJID : official publication of the International Society for Infectious Diseases 2015; 30: 67-73.

32 Pallin DJ, Binder WD, Allen MB et al. Clinical trial: comparative effectiveness of cephalexin plus trimethoprim-sulfamethoxazole versus cephalexin alone for treatment of uncomplicated cellulitis: a randomized controlled trial. Clinical infectious diseases : an official publication of the Infectious Diseases Society of America 2013; 56: 1754-62.

33 Chan JC. Ampicillin/sulbactam versus cefazolin or cefoxitin in the treatment of skin and skin-structure infections of bacterial etiology. Advances in therapy 1995; 12: 139-46.

34 Wilcox MH, Corey GR, Talbot GH et al. CANVAS 2: the second Phase III, randomized, double-blind study evaluating ceftaroline fosamil for the treatment of 
patients with complicated skin and skin structure infections. The Journal of antimicrobial chemotherapy 2010; 65 Suppl 4: iv53-iv65.

35 Daniel R. (Study B: azithromycin vs cloxacillin). Azithromycin, erythromycin and cloxacillin in the treatment of infections of skin and associated soft tissues. European Azithromycin Study Group. The Journal of international medical research 1991; 19: 433-45.

36 Grayson ML, McDonald M, Gibson K et al. Once-daily intravenous cefazolin plus oral probenecid is equivalent to once-daily intravenous ceftriaxone plus oral placebo for the treatment of moderate-to-severe cellulitis in adults. Clinical infectious diseases : an official publication of the Infectious Diseases Society of America 2002; 34: 1440-8.

37 Corey GR, Wilcox MH, Talbot GH et al. CANVAS 1: the first Phase III, randomized, double-blind study evaluating ceftaroline fosamil for the treatment of patients with complicated skin and skin structure infections. The Journal of antimicrobial chemotherapy 2010; 65 Suppl 4: iv41-51.

38 Corey GR, Good S, Jiang H et al. Single-dose oritavancin versus 7-10 days of vancomycin in the treatment of gram-positive acute bacterial skin and skin structure infections: the SOLO II noninferiority study. Clinical infectious diseases : an official publication of the Infectious Diseases Society of America 2015; 60: 254-62.

39 Giordano P, Song J, Pertel P et al. Sequential intravenous/oral moxifloxacin versus intravenous piperacillin-tazobactam followed by oral amoxicillinclavulanate for the treatment of complicated skin and skin structure infection. International journal of antimicrobial agents 2005; 26: 357-65.

40 Weigelt J, Itani K, Stevens D et al. Linezolid versus vancomycin in treatment of complicated skin and soft tissue infections. Antimicrobial agents and chemotherapy 2005; 49: 2260-6.

41 Kiani R. Double-blind, double-dummy comparison of azithromycin and cephalexin in the treatment of skin and skin structure infections. European journal of clinical microbiology \& infectious diseases : official publication of the European Society of Clinical Microbiology 1991; 10: 880-4.

42 Thomas MG. Oral Clindamycin Compared With Sequential Intravenous and Oral Flucloxacillin in the Treatment of Cellulitis in Adults: A Randomized, Double-Blind Trial. Infectious Diseases in Clinical Practice 2014; 22: 330-4.

43 Vinen J, Hudson B, Chan B et al. A Randomised Comparative Study of Once-Daily Ceftriaxone and 6-Hourly Flucloxacillin in the Treatment of Moderate to Severe Cellulitis. Clinical Drug Investigation 1996; 12: 221-5.

44 Boucher HW, Wilcox M, Talbot GH et al. Once-weekly dalbavancin versus daily conventional therapy for skin infection. The New England journal of medicine 2014; 370: 2169-79.

45 Hepburn MJ, Dooley DP, Skidmore PJ et al. Comparison of short-course (5 days) and standard (10 days) treatment for uncomplicated cellulitis. Archives of internal medicine 2004; 164: 1669-74.

46 Moran GJ, Fang E, Corey GR et al. Tedizolid for 6 days versus linezolid for 10 days for acute bacterial skin and skin-structure infections (ESTABLISH-2): a randomised, double-blind, phase 3, non-inferiority trial. The Lancet Infectious Diseases 2014; 14: 696-705.

47 DiMattia AF, Sexton MJ, Smialowicz CR et al. Efficacy of two dosage schedules of cephalexin in dermatologic infections. The Journal of family practice 1981; 12: 649-52.

48 Iannini PB, Kunkel MJ, Link J, Arthur Stanley et al. Multicenter Comparison of Cefonicid and Cefazolin in Hospitalized Patients with Skin and Soft Tissue Infections. Advances in therapy 1985; 2: 214-24.

49 Schwartz R, Das-Young LR, Ramirez-Ronda $C$ et al. Current and future management of serious skin and skin-structure infections. The American journal of medicine 1996; 100: 90S-5S. 
50 Covington P, Davenport J M, Andrae D et al. Randomized, double-blind, phase II, multicenter study evaluating the safety/tolerability and efficacy of J NJ-Q2, a novel fluoroquinolone, compared with linezolid for treatment of acute bacterial skin and skin structure infection. Antimicrobial agents and chemotherapy 2011; 55: 57907.

51 Daniel R. (Study A: azithromycin vs erythromycin). Azithromycin, erythromycin and cloxacillin in the treatment of infections of skin and associated soft tissues. European Azithromycin Study Group. The Journal of international medical research 1991; 19: 433-45.

52 Miller LG, Daum RS, Creech CB et al. Clindamycin versus trimethoprimsulfamethoxazole for uncomplicated skin infections. The New England journal of medicine 2015; 372: 1093-103.

53 Noel GJ, Strauss RS, Amsler K et al. Results of a double-blind, randomized trial of ceftobiprole treatment of complicated skin and skin structure infections caused by gram-positive bacteria. Antimicrobial agents and chemotherapy 2008; 52: 37-44.

54 Noel GJ, Bush K, Bagchi P et al. A randomized, double-blind trial comparing ceftobiprole medocaril with vancomycin plus ceftazidime for the treatment of patients with complicated skin and skin-structure infections. Clinical infectious diseases : an official publication of the Infectious Diseases Society of America 2008; 46: 647-55.

55 Bucko AD, Hunt BJ, Kidd SL et al. Randomized, double-blind, multicenter comparison of oral cefditoren 200 or $400 \mathrm{mg}$ BID with either cefuroxime $250 \mathrm{mg}$ BID or cefadroxil $500 \mathrm{mg}$ BID for the treatment of uncomplicated skin and skinstructure infections. Clinical therapeutics 2002; 24: 1134-47.

56 Fabian TC, File TM, Embil J M et al. Meropenem versus imipenem-cilastatin for the treatment of hospitalized patients with complicated skin and skin structure infections: results of a multicenter, randomized, double-blind comparative study. Surgical infections 2005; 6: 269-82.

57 Kauf TL, McKinnon P, Corey GR et al. An open-label, pragmatic, randomized controlled clinical trial to evaluate the comparative effectiveness of daptomycin versus vancomycin for the treatment of complicated skin and skin structure infection. BMC infectious diseases 2015; 15: 503.

58 Leman P, Mukherjee D. Flucloxacillin alone or combined with benzylpenicillin to treat lower limb cellulitis: a randomised controlled trial. Emergency medicine journal : EMJ 2005; 22: 342-6.

59 Tack KJ, Littlejohn TW, Mailloux G et al. Cefdinir versus cephalexin for the treatment of skin and skin-structure infections. The Cefdinir Adult Skin Infection Study Group. Clinical therapeutics 1998; 20: 244-56.

60 Pertel PE, Eisenstein BI, Link AS et al. The efficacy and safety of daptomycin vs. vancomycin for the treatment of cellulitis and erysipelas. International journal of clinical practice 2009; 63: 368-75.

61 Prince WT, I vezic-Schoenfeld Z, Lell C et al. Phase II clinical study of BC-3781, a pleuromutilin antibiotic, in treatment of patients with acute bacterial skin and skin structure infections. Antimicrobial agents and chemotherapy 2013; 57: 2087-94.

62 Prokocimer $\mathrm{P}$, De Anda C, Fang E et al. Tedizolid phosphate vs linezolid for treatment of acute bacterial skin and skin structure infections: the ESTABLISH-1 randomized trial. Jama 2013; 309: 559-69.

63 Rao B, See RC, Chuah SK et al. Ticarcillin plus clavulanic acid versus moxalactam in the treatment of skin and soft tissue infections. The American journal of medicine 1985; 79: 126-9.

64 Saches MK, Pilgrim C. Ampicillin/Sulbactam Compared with Cefazolin or Cefoxitin for the Treatment of Skin and Skin Structure Infections. Drug Invest. 1990; 2: 173-83.

65 Zeglaoui F, Dziri C, Mokhtar I et al. Intramuscular bipenicillin vs. intravenous penicillin in the treatment of erysipelas in adults: randomized controlled study. Journal of the European Academy of Dermatology and Venereology : JEADV 2004; 18: 426-8. 
66 Aboltins CA, Hutchinson AF, Sinnappu RN et al. Oral versus parenteral antimicrobials for the treatment of cellulitis: a randomized non-inferiority trial. The Journal of antimicrobial chemotherapy 2015; 70: 581-6.

67 Bergkvist P-i, Sjöbeck K. Antibiotic and Prednisolone Therapy of Erysipelas: A Randomized, Double Blind, Placebo-controlled Study. Scandinavian Journal of Infectious Diseases 2009; 29: 377-82.

68 Baig A, Grillage MG, Welch RB. A comparison of erythromycin and flucloxacillin in the treatment of infected skin lesions in general practice. Br J Clin Pract 1988; 42: $110-5$.

69 Johnson S, Leak K, Singh S et al. Can cycloidal vibration plus standard treatment reduce lower limb cellulitis treatment times? Journal of wound care 2007; 16: 166-9.

\section{Results Tables}

Table 1. Categorisation of clinical response and timing of assessment 
Cure, failure

Cure, improvement, failure, indeterminate

Cure, failure, indeterminate

Cure, improvement, failure

Success, failure

Relapse/ recurrence

Satisfactory, unsatisfactory

Cured, not cured

Cure, failure, not evaluable

Cure + lesion regression

Cure, failure, relapse

Satisfactory (cure), unsatisfactory (failure/relapse)

Cure, recurrence

Clinical success (cure, improvement), no improvement, failure Failure

Successful response, stable response, failure

Success (cure/ improvement), failure, indeterminate/ not assessable Cure, improvement

Success, improvement, failure

Responder, non-responder

Responder, non-responder, indeterminate

Responder, failure

Satisfactory, failure, indeterminate, unevaluable

Cure, improvement, failure, relapse

Failure, recovery

Variable ${ }^{28}$

Day 25-35 (follow-up) ${ }^{29}$

Day 7-14 after EOT (TOC) ${ }^{30}$

Day 14-21 after EOT (TOC) ${ }^{31}$

1 month after EOT $^{32}$

Unclear ${ }^{33}$

Day 7 after EOT $^{34}$

Day 4-9 after EOT ${ }^{35}$

EOT (variable) ${ }^{36}$

Day 1 after EOT, Day 8-15 after EOT (TOC) $)^{37} 4$

Day 7-14 after EOT $^{38}$

Day 10-42 after EOT (TOC) ${ }^{39}$

EOT, Day 8-15 after EOT (TOC) ${ }^{40}$

Day 11 after treatment initiation ${ }^{41}$

EOT (variable) 42

48-72 hrs after completion of IV therapy 43

48-72hrs after treatment initiation, Day 14-15 3

(EOT) 44

Day 14, Day 28 after treatment initiation ${ }^{45}$

Day 7 after treatment initiation ${ }^{46}$

21-35 days after EOT $^{37,40}$

Day 28-35 after EOT (TOC) ${ }^{31}$

Unclear ${ }^{42}$

Unclear $^{47,48,49} 3$

Day 10-14 after treatment initiation (TOC), 2- 3

14 days after EOT (short-term follow-up) ${ }^{50}$

Day 4-11 after EOT 51

Day 12 (EOT), Day 7-10 after EOT, Day 40 (1

month follow-up) ${ }^{52}$

Day 7-14 after EOT (TOC) 53,54

Day 28-35 after EOT (TOC) $)^{54}$

Day 14-17 (EOT) ${ }^{29}$

Day 7-14 after EOT (TOC) ${ }^{55}$

Within 24 hrs last dose (EOT), Day 7-14 after 1 $\mathrm{EOT}^{56}$

1 month after EOT $^{36}$

48-72 hr after treatment initiation ${ }^{57}$

1

After 24 hrs of treatment ${ }^{58}$

1

48-72 hr after treatment initiation ${ }^{46}$

1

Day 11 (EOT), Day 7-14 after EOT ${ }^{46}$

5

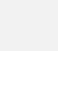


i) absence of fever ii) no increase in area of erythema plus absence of fever iii) no increase in area of erythema, no increase in area of swelling and the absence of fever iv) $>20 \%$ reduction in area of erythema Resolution of symptoms other than slight residual erythema/oedema Clinical signs and symptoms resolved with no evidence of active infection at the time treatment was discontinued and no evidence of relapse

(i) Complete resolution of signs and symptoms of soft-tissue infection that was sufficient enough to result in either discontinuation of all antibiotic therapy or switch to the use of oral agents (ii) with no recurrence at the same site in 1 month

All signs and symptoms of the infection that were present before therapy were improved or had resolved and no new signs or symptoms of the infection were present at the post-treatment follow-up

Days until no advancement of the area of cellulitis

Days until no remaining flush/elevation of body temperature $>37.8$ degrees Celsius on that day

A score of 0 for erythema, oedema and pain and a normal temperature

Time to resolution of erythema, oedema, pain and temperature, without additional antibiotics

Body temperature $<37.5$ degrees, complete regression of local/general signs of severity and disappearance of the cutaneous plaque

Lesion size, defined as its length times its width, decreased from baseline, temperature was $\leq 37.6^{\circ} \mathrm{C}$, fluctuance and localized heat/warmth were absent, and tenderness to palpation and swelling/induration were no worse than mild; for patients with a wound infection, the purulent drainage was to be improved and no worse than mild

Disappearance of warmth and tenderness at the site of infection, with substantial improvement in erythema and oedema, even with mild residual erythema, hyperpigmentation or oedema, not requiring further antibiotic therapy at day 14, and without symptom recurrence at day 28

Signs and symptoms disappeared or improved or signs and symptoms disappeared or improved during therapy but recurred

Improvement in signs and symptoms $1^{63}$

Not defined $2^{52,60}$

\pm Outcomes in line with FDA guidance: cessation of lesion spread (measured as the product of the length $x$ width of lesion) $48-72 \mathrm{hr}$ after treatment initiation

Table 3. Method of assessment of patient-reported pain Method of assessment Trials

$0=$ absent,$+/ 1=$ moderate,$++/ 2=$ marked/severe $\quad 3^{28,29,65}$

$100 \mathrm{~mm}$ VAS scalet $\quad 3^{46,58,60}$ 


\begin{tabular}{ll}
\hline 10cm VAS scale \pm & $1^{66}$ \\
\hline Faces rating scale \pm & $1^{46}$ \\
\hline Severity rating: none, mild, moderate, severe & $1^{68}$ \\
\hline Likert pain scale $(0-10)$ & $1^{45}$ \\
\hline McGill pain score & $1^{69}$ \\
\hline Brief pain Inventory & $1^{57}$ \\
\hline $0=$ "no pain" and the $100=$ "worst pain". \pm 10 point scale. $0=$ "no pain" and $10=$ "worst pain you can \\
imagine"
\end{tabular}

Table 4. Categorisation of microbial outcomes 


\begin{tabular}{|c|c|c|}
\hline Complete eradication & $\begin{array}{l}\text { Absence of culturable material/pre } \\
\text { treatment pathogen from the original } \\
\text { infection site by the end of therapy }\end{array}$ & $\begin{array}{c}17^{30,31,33,35-37,39,41,46,49,51,53-} \\
55,59,63,64\end{array}$ \\
\hline & Not defined & $3^{50,60,61}$ \\
\hline \multirow[t]{2}{*}{ Presumed eradication } & $\begin{array}{l}\text { Adequate source specimen not available } \\
\text { to culture but patient was assessed as a } \\
\text { clinical cure/responded }\end{array}$ & $9^{30,31,37,39,46,53,54,59}$ \\
\hline & Not defined & $3^{50,60,61}$ \\
\hline Partial eradication & $\begin{array}{l}\text { Absence of some, but not all pre- } \\
\text { treatment pathogens at the end of } \\
\text { therapy }\end{array}$ & $2^{33,64}$ \\
\hline Success & $\begin{array}{l}\text { Documented/presumed eradication of } \\
\text { the baseline pathogen }\end{array}$ & $1^{34}$ \\
\hline \multirow[t]{2}{*}{ Cure } & $\begin{array}{l}\text { The pathogen was eliminated with a } \\
\text { clearing of inflammation, or the infection } \\
\text { recurred with a new pathogen }\end{array}$ & $1^{47}$ \\
\hline & $\begin{array}{l}\text { Eradication of causative pathogen or no } \\
\text { materials for culture due to clinical } \\
\text { success }\end{array}$ & $1^{43}$ \\
\hline \multirow[t]{4}{*}{ Persistence } & $\begin{array}{l}\text { Growth of the pre-treatment pathogen in } \\
\text { a culture taken at the post-treatment } \\
\text { visit }\end{array}$ & $12^{30,31,33,36,39,47,49,53,55,59,63,64}$ \\
\hline & $\begin{array}{l}\text { Absence of appropriate culture material } \\
\text { in a clinical failure }\end{array}$ & $1^{39}$ \\
\hline & $\begin{array}{l}\text { Eradication of original pathogen with a } \\
\text { post-baseline positive culture with a new } \\
\text { pathogen requiring treatment }\end{array}$ & $1^{39}$ \\
\hline & Not defined & $8^{34,35,37,46,50,51,54,61}$ \\
\hline \multirow[t]{2}{*}{ Presumed persistence } & $\begin{array}{l}\text { No pathogen identified in a patient who } \\
\text { was assessed as clinical failure }\end{array}$ & $3^{30,31,53}$ \\
\hline & Not defined & $4^{46,50,54,61}$ \\
\hline \multirow[t]{3}{*}{$\begin{array}{l}\text { Superinfection/ new } \\
\text { infection }\end{array}$} & $\begin{array}{l}\text { Emergence of a different pathogen } \\
\text { organism during or at the end of therapy }\end{array}$ & $3^{30,33,36}$ \\
\hline & $\begin{array}{l}\text { Pathogen different from that isolated at } \\
\text { baseline in presence of signs/symptoms } \\
\text { of infection }\end{array}$ & $3^{31,53,59}$ \\
\hline & Not defined & $3^{50,54,61}$ \\
\hline \multirow[t]{2}{*}{ Failure } & $\begin{array}{l}\text { Lesion persisted with significant numbers } \\
\text { of the original pathogen, or lesion } \\
\text { improved but recurred with a culture } \\
\text { that was positive with the same } \\
\text { pathogen }\end{array}$ & $1^{47}$ \\
\hline & $\begin{array}{l}\text { Persistence/ presumed persistence of } \\
\text { causative pathogen }\end{array}$ & $2^{40,43}$ \\
\hline Recurrence & $\begin{array}{l}\text { Initial clearance of pre-treatment } \\
\text { pathogen, same pathogen reappeared } \\
\text { during the follow-up period }\end{array}$ & $1^{55}$ \\
\hline Colonisation & $\begin{array}{l}\text { Pathogen isolated in absence of clinical } \\
\text { signs/ symptoms of infection }\end{array}$ & $1^{53}$ \\
\hline $\begin{array}{l}\text { I ndeterminate/ not } \\
\text { evaluable }\end{array}$ & $\begin{array}{l}\text { Unevaluable results/ response not fitting } \\
\text { any other category }\end{array}$ & $11^{33,34,37,39,40,43,53,54,59,61,64}$ \\
\hline
\end{tabular}





\section{Figures}

Figure 1. Broad outcome domains used across trials

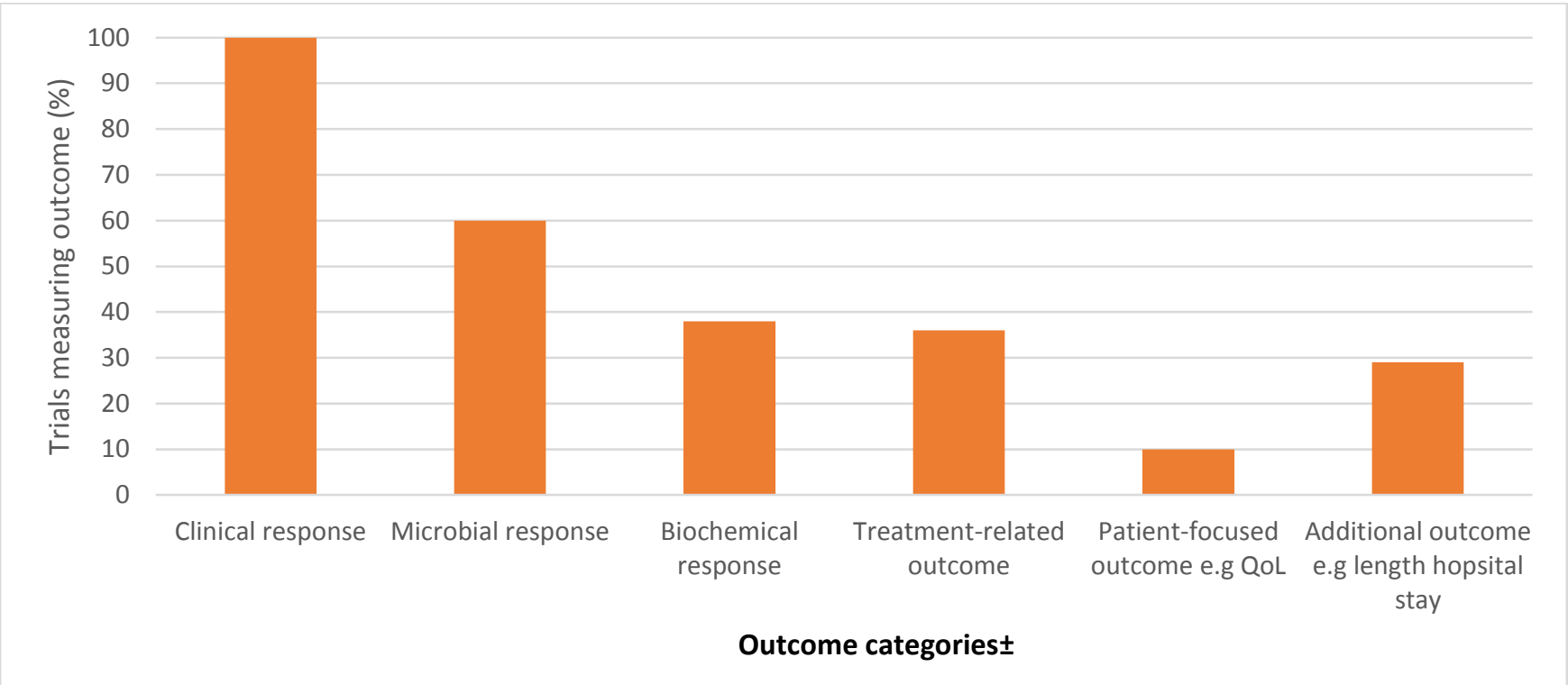

\pm Explanation of outcome categories. Clinical: signs and symptoms of infection assessed as a measure of treatment effect, Microbial: growth or elimination of organisms at the site of infection or in blood cultures as a measure of treatment effect, Biochemical: blood markers measured before and after treatment, Treatmentrelated: e.g number of doses/ length of antibiotic therapy used as an outcome, Patient-focused: e.g QoL effects in response to treatment 
Figure 2. Signs and symptoms of infection assessed in clinical response

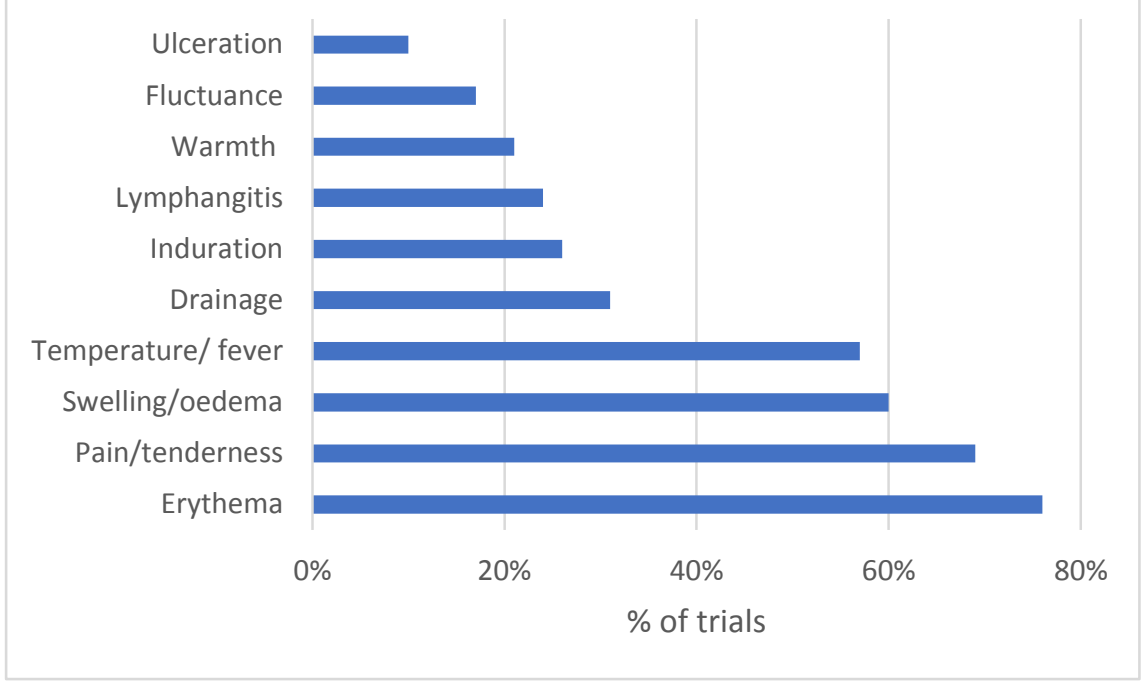


Figure 3. Method of assessment of erythema

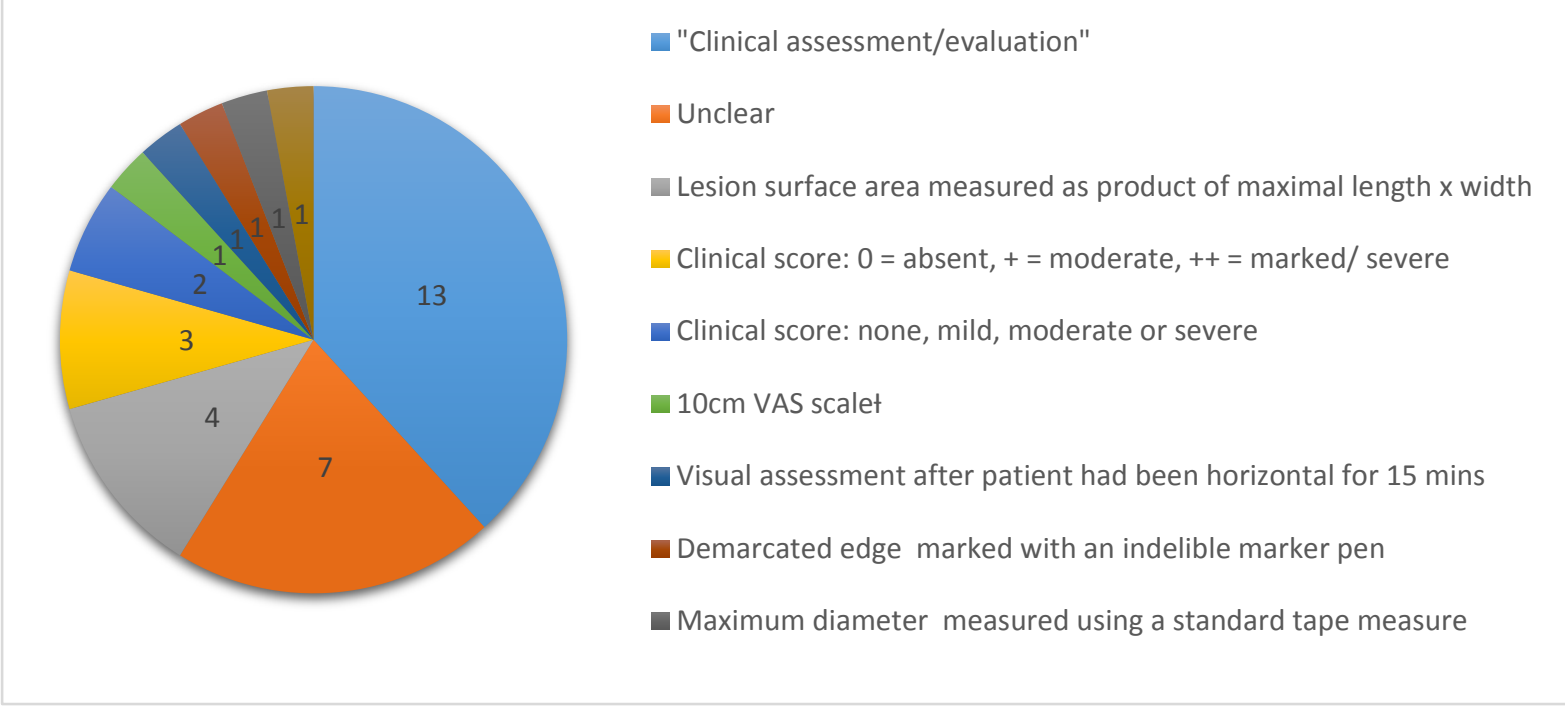

$+10 \mathrm{~cm}$ VAS scale: ranging from skin normal colour, no inflammation at 0.0 to skin very red and inflamed at 10.0 
Figure 4. Outcomes themes of importance extracted from participant feedback in the Cellulitis Priority-setting Partnership

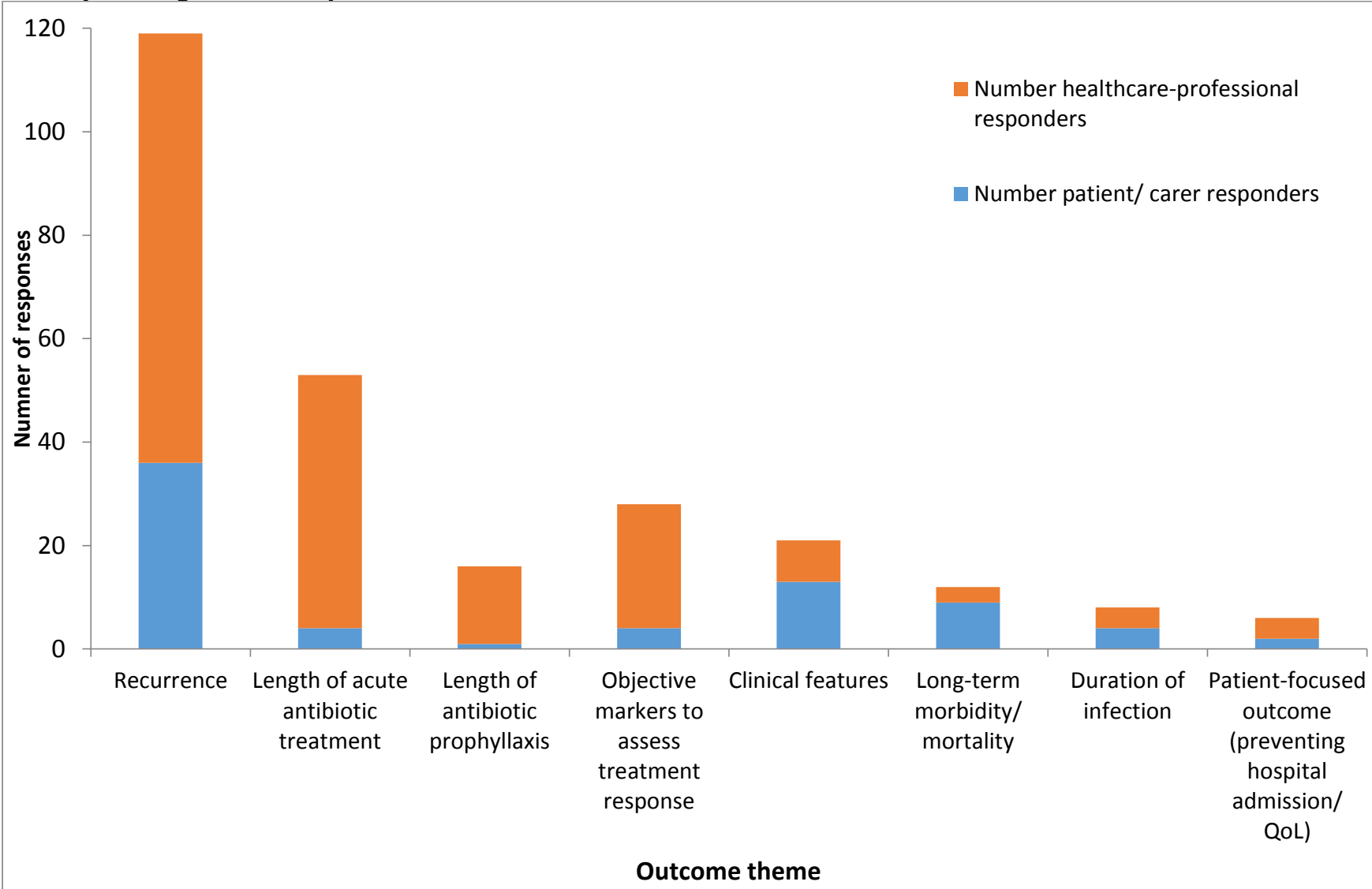

